University of Wollongong

Research Online

SMART Infrastructure Facility - Papers

Faculty of Engineering and Information

Sciences

2007

\title{
Towards an Integrated Model of Enterprise Systems
}

Grace A. Kennedy

University of Wollongong, gracek@uow.edu.au

C E. Siemieniuch

Loughborough University

M A. Sinclair

Loughborough University

Follow this and additional works at: https://ro.uow.edu.au/smartpapers

Part of the Engineering Commons, and the Physical Sciences and Mathematics Commons

Research Online is the open access institutional repository for the University of Wollongong. For further information contact the UOW Library: research-pubs@uow.edu.au 


\title{
Towards an Integrated Model of Enterprise Systems
}

\begin{abstract}
An enterprise system consists of a number of components or building blocks. It is common to use views or models of the enterprise that contain a selection of these components (dependent on the intended usage of the model). The premise is that if these views are considered systems in their own right then the total enterprise system is actually a system-of-systems. Difficulty arises however when the boundaries between the systems overlap - it is therefore necessary to have an integrated model of the total enterprise that can cope with these overlaps and hence interactions between the systems. Within this paper there will be two main areas of work described; firstly the development of models/tools of "soft" enterprise characteristics; and secondly how these characteristics may be included in an integrated model of an enterprise system. Case studies of UK organisations (primarily within the defence industry) were undertaken to provide context to the results.
\end{abstract}

\section{Keywords}

systems, towards, enterprise, model, integrated

Disciplines

Engineering | Physical Sciences and Mathematics

\section{Publication Details}

Kennedy, G. A. L., Siemieniuch, C. E. \& Sinclair, M. A. (2007). Towards an Integrated Model of Enterprise Systems. INCOSE International Symposium, 17 (1), 1256-1274. 


\title{
Towards an Integrated Model of Enterprise Systems
}

\author{
G.A.L. Kennedy, C.E. Siemieniuch and M.A. Sinclair \\ Loughborough University \\ Systems Engineering Innovation Centre (SEIC), Holywell Park, Loughborough University, \\ Ashby Road, Loughborough, Leicestershire. LE11 3TU. UK \\ Corresponding author: G.A.L.Kennedy@lboro.ac.uk \\ Copyright @ 2007 by G.A.L. Kennedy. Published and used by INCOSE with permission.
}

\begin{abstract}
An enterprise system consists of a number of components or building blocks. It is common to use views or models of the enterprise that contain a selection of these components (dependent on the intended usage of the model). The premise is that if these views are considered systems in their own right then the total enterprise system is actually a system-of-systems. Difficulty arises however when the boundaries between the systems overlap - it is therefore necessary to have an integrated model of the total enterprise that can cope with these overlaps and hence interactions between the systems. Within this paper there will be two main areas of work described; firstly the development of models/tools of "soft" enterprise characteristics; and secondly how these characteristics may be included in an integrated model of an enterprise system. Case studies of UK organisations (primarily within the defence industry) were undertaken to provide context to the results.
\end{abstract}

\section{Introduction}

The Problem/Background. Vernadat defines the term enterprise as a socio-economic organisation created to produce products or procure services and to make a profit. He goes on to explain that enterprise modelling is the process of building models of the whole or parts of an enterprise from knowledge about the enterprise, previous models, and/or reference models as well as domain ontologies and model representation languages (Vernadat 1996).

Organisational design theory emerged in the early 1950s (Katz 1970). The theory behind organisational behaviour has had to adapt and extend as organisations and their products have become more complex and presented more emergent problems - for example take the boom in information technology and distributed organisations. Enterprise modelling has been used to understand and analyse organisations. Much work has occurred in the last ten years into providing a standard framework for enterprise modelling in order to provide interoperability between organisations (Chen \& Vernadat 2004).

It is heard in the news that companies are dynamically restructuring in order to improve their organisational performance in terms of time, cost and quality. There are often problems with this restructuring which result in money and time being wasted as well as loss of motivation/morale of employees due to these changes. Possible explanations could be:

- The theory being used only worked on previous configurations of the organisation

- The theory used is not contextual or applicable to their current enterprise situation

- The process by which change is implemented may not have been considered fully 
- Many analysts of organisations do not consider the total enterprise as a system (e.g. they try to improve one part but do not consider the impact on other parts of the system)

It is this last point that has driven a project at Loughborough University. The Virtual Organisational Rig for Testing and Investigating Company Structures (VORTICS) project lasted 30 months and was completed in late 2006. VORTICS was set up as a feasibility study to assess whether an integrated software rig for testing organisations could be developed and specified.. The objectives of the project were to:

i. $\quad$ capture the requirements for enterprise modelling (EM)

ii. undertake a literature review into the current capabilities of EM

iii. perform a gap analysis between the two former points

iv. develop and refine models and tools to cover these gaps

v. integrate new and traditional models

vi. develop a specification for the VORTICS

It is objectives $i v$ and $v$ that will be covered in more detail in this paper. New models and tools were developed for a set of "soft" or human aspects of the enterprise. The relationships between components of these models and more traditional forms of enterprise modelling were identified to create the integrated enterprise system model.

Section Breakdown/Structure. The first section of this paper introduces the views/models within enterprises and the enterprise as a systems-of-systems. The second section provides an overview of the gaps within enterprise modelling in terms of "soft" or human aspects and the tools/models Loughborough University have developed in answer to these needs. The third section introduces an integrated model of the enterprise system including both the traditional forms of enterprise modelling and the models described in the previous section. The final section concludes the paper and discusses the usefulness of the integrated model.

\section{The Enterprise as a System-of-Systems}

The Generic Enterprise Reference Architecture (GERA) within the methodology of the same name (GERAM) describes a framework for different aspects of modelling an enterprise. It does not specify the actual models that are required but provides an empty shell to show what can be modelled. GERAM was standardized as Annex A of ISO15704 (IFIP/IFAC 2000). Figure 1 shows the GERA cube.

As with most enterprise architectures, it is not essential for every cube within the GERA framework to be populated for enterprise modelling. A subset of cubes should be selected that best gives the information required to solve the enterprise problem/issue being addressed.

There are 3 axes on the GERA cube;

1. Views - GERA specifies that there are four views within an enterprise. The functional view (covers processes), the information view (mainly information flows and supporting infrastructure), the organisational view (traditionally this is the domain of organisational charts), and the resource view (human and technological resource management).

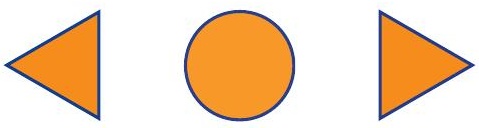


2. Lifecycle - The model of a view of an enterprise will differ depending on the stage in the development lifecycle the enterprise system has reached.

3. Instantiation - There are different levels of genericity that a model can be presented in. Particular models only apply to a specific organisation, partial models apply to similar scenarios (e.g. models of organisations in the same domain or with similar goals), and generic models can be applied and tailored to any organisation.

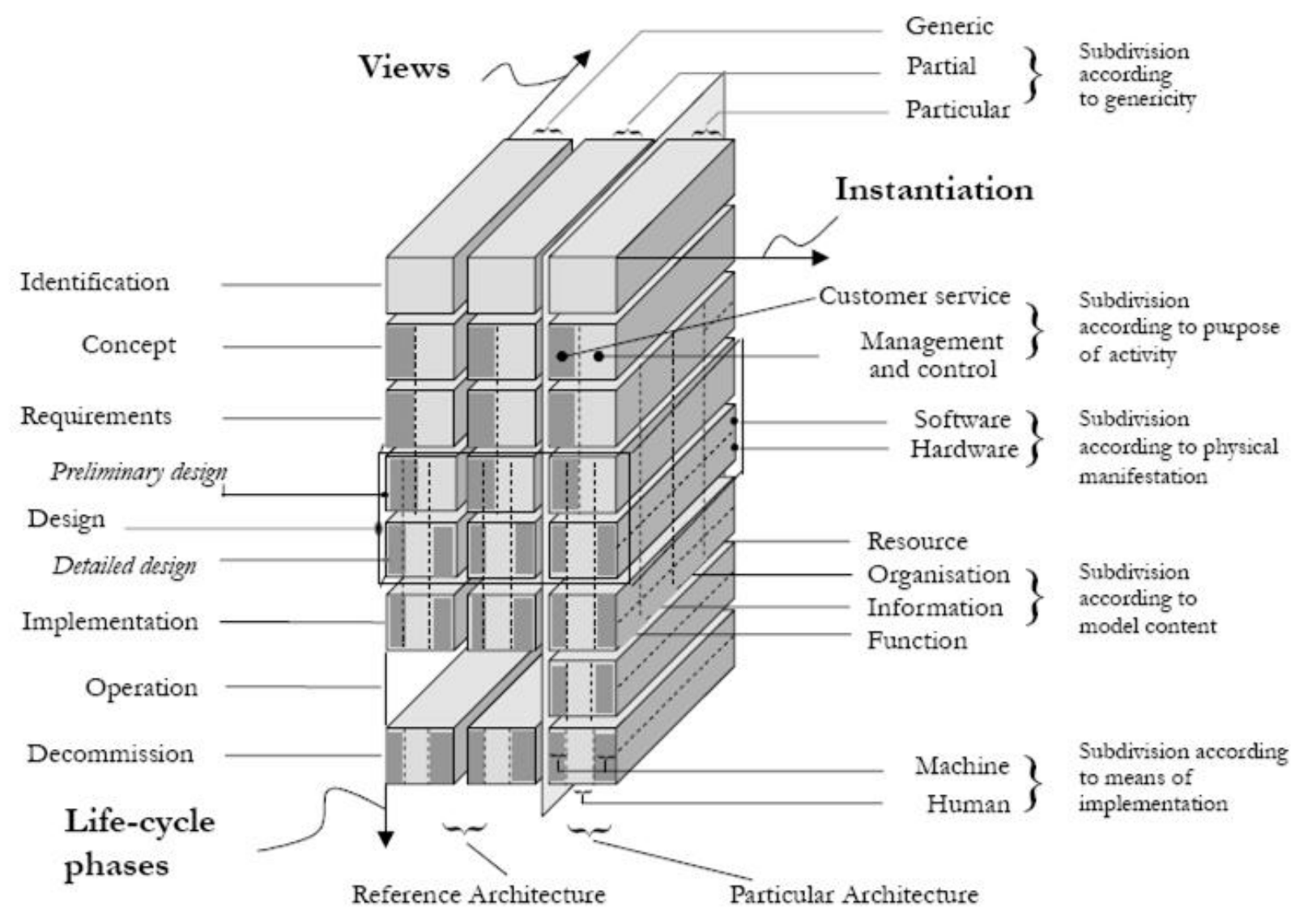

Figure 1. Generic Enterprise Reference Architecture (GERA)

An enterprise system consists of a number of components or building blocks. It is common to use views or models of the enterprise that contain a selection of these components (dependent on the intended usage of the model). The premise is that if these views are considered systems in their own right then the total enterprise system is actually a system-ofsystems. Using the four GERA views a number of components of the enterprise can be grouped. Figure 2 shows a visualisation of the enterprise system-of-systems, it is not totally exhaustive in terms of the components or links identified but seeks to illustrate that the systems are not totally independent rather that there are links between some of the components, resulting in a closely coupled system. The block arrow lines show where a component may actually be the same component, dotted arrow lines show a relationship between components. 


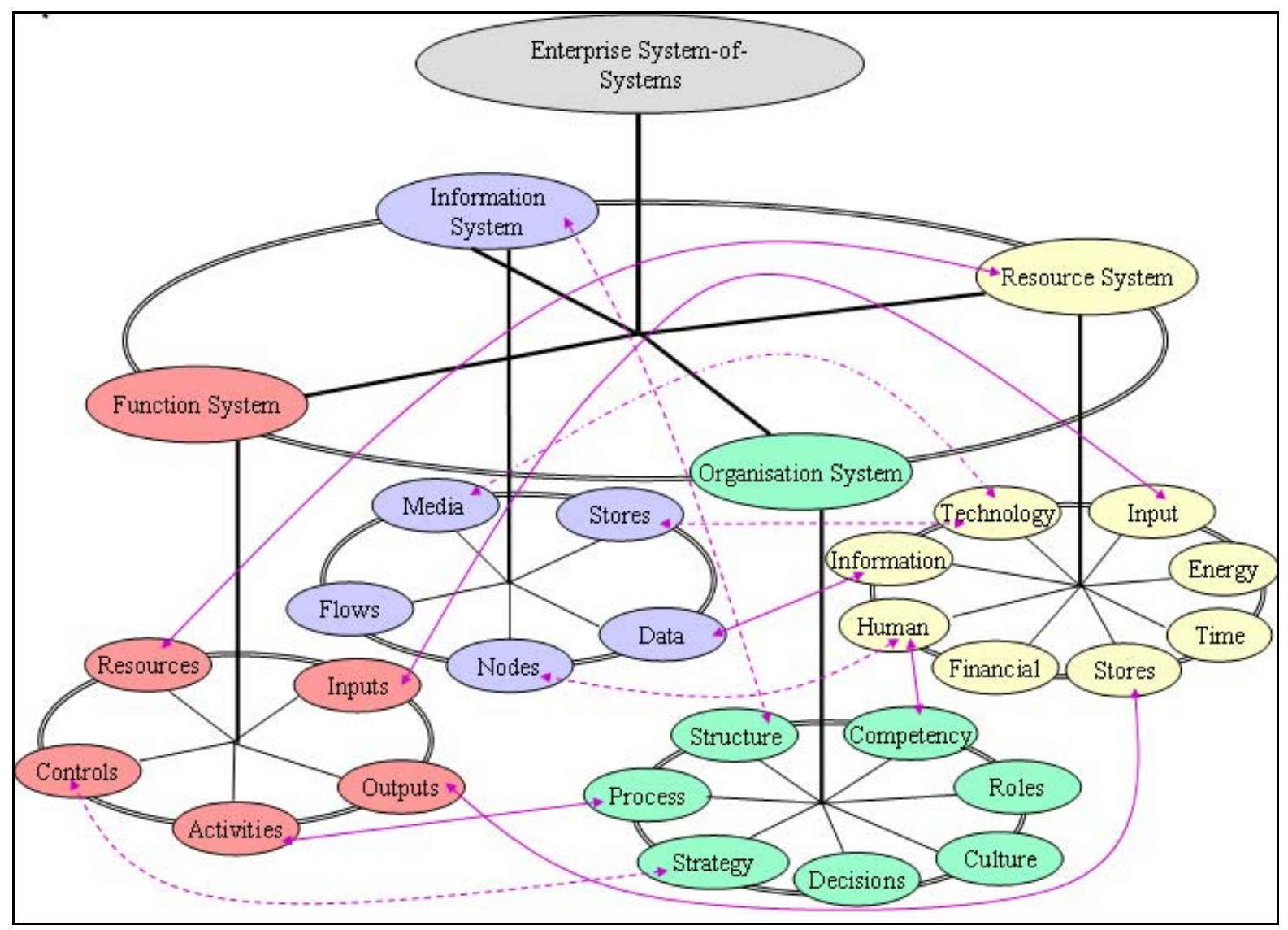

Figure 2. The Enterprise as a System-of-Systems

\section{Soft Enterprise Characteristics}

In addition to the more traditional forms of enterprise modelling (functional/process, information, organisational structure and resources) a set of soft enterprise characteristics were identified based on the results from a gap analysis between current enterprise modelling capability and requirements for enterprise modelling. The following enterprise characteristics were investigated and modelled using the tools created/developed by LU; Roles, Cultural Values, Enterprise Strategy, Decision Making Systems and Competency Requirements. An organisation can be defined as a socio-grouping working towards a common goal. It was decided to expand the organisational view of the enterprise from being mostly structure and hierarchy based to include these additional soft/human aspects (see figure 2).

These models/tools were in various stages of maturity and case study scenarios were used to provide "real data" which could be used to improve and validate the prototype tools. Three scenarios were modelled;

- Scenario A covered the engineering organisation on a project within a large engineering systems company. The purpose of modelling Scenario A was to provide input into internal auditing activities. 
- Scenario B covered the extended enterprise involved in the delivery of defence capability through the smart acquisition process. The purpose of modelling Scenario $\mathrm{B}$ was to highlight the value of enterprise modelling through providing insight into problems/issues with the relationships between the different suborganisations.

- Scenario C covered a research organisation within a university, in particular the suborganisation of a collaborating industrial partner. The purpose of modelling Scenario $\mathrm{C}$ was to identify and highlight the organisational issues (specifically the soft enterprise characteristics problems).

Role Modelling. Roles are a subset of organisational structure: they can be held by human or technical agents as individuals or as a group. They are the key resource that ensures a function or process is carried out. An individual agent may hold one or many roles and one or many roles for human agents may be combined to form a job.

The roles interactions were modelled using the Role Matrix Technique (RMT), developed by Loughborough University (Callan et. al. 2005). The RMT has two main stages that build upon the process model of an organisation. Firstly the process model is expanded to identify which roles are involved in each activity, and nature of that involvement. This is done using the Cross Hair diagram (see figure 3). A role positioned in the top left hand quadrant is the role in control of the activity (there should only be ONE role in this quadrant at any one time). The bottom left quadrant may hold any roles that actually execute or implement the activity. The top right quadrant holds roles that provide constraining advice (i.e. the advice that the controller or executer must listen to). The bottom right quadrant holds roles that provide discretionary advice (i.e. the advice that is optional to take heed of).

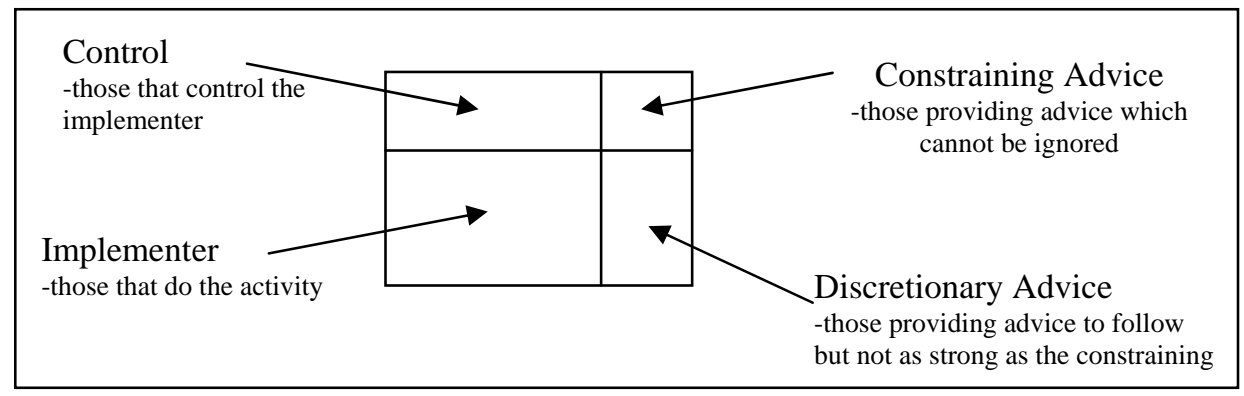

Figure 3. Role Cross Hair Notation

The RMT compliments the IDEF0 notation and is decomposable (NIST 1993). In these cases the roles can be amalgamated from the bottom up to show the overall roles and their most likely position in the quadrant.

The second stage of the RMT utilises the role matrix. The roles are translated from the crosshair boxes onto the matrix. The vertical axis represents the degree to which the role has in planning their own activities; the horizontal axis represents the degree to which the role has in how they execute their activities. The position on the matrix is relative to the other roles involved. Figure 4 shows the key for the different types of interaction. Figure 5 shows an example of a populated role matrix for the baseline process with amalgamated views from the different roles involved. 


\begin{tabular}{|l|l|}
\hline$\longleftarrow$ & Handover \\
\hline$\downarrow$ & Delegation \\
\hline$-\rightarrow$ & $\begin{array}{l}\text { Constraining Advice } \\
\text { (red dashdot) }\end{array}$ \\
\hline$\ldots \ldots$ & $\begin{array}{l}\text { Discretionary Advice } \\
\text { (black dashed) }\end{array}$ \\
\hline
\end{tabular}

Figure 4. Communications Key for the Role Matrix

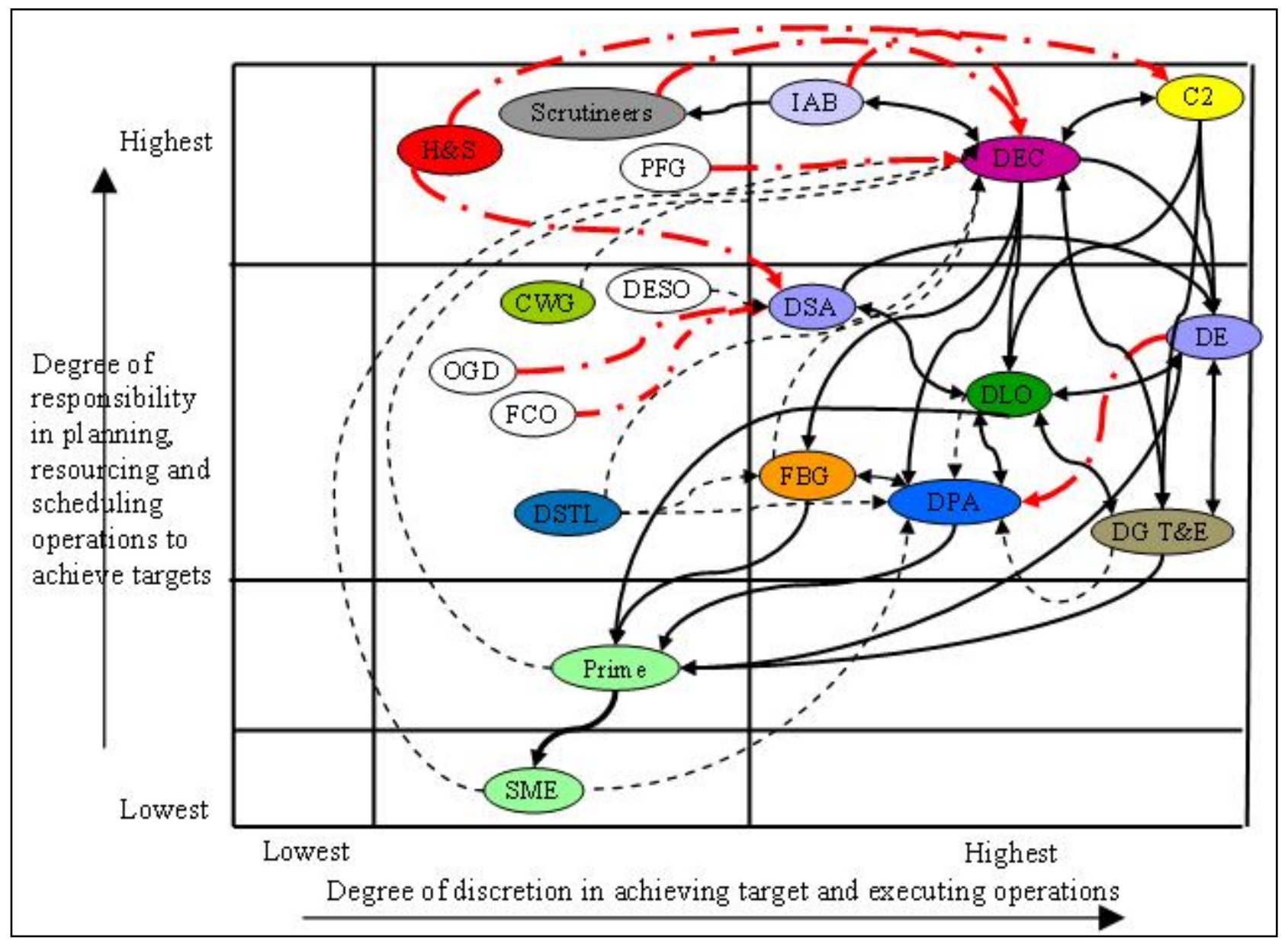

Figure 5. Role Matrix Model for Scenario B

The RMT provides on overview for the organisation. It enables the viewer to see the general roles involved in the process and their positions relative to each other as well as the lines of responsibility (in the example in figure 5 there is a definite flow of control from top right to bottom left). The process by which the RMT is used also highlights differences in viewpoints of the roles involved and the nature of that relationship. It can also be indicative of whether there are bottlenecks or workload issues (e.g. if there are many relationships to/from a role). 
Cultural Values Identification. The term culture refers to "the collective programming of the mind which distinguishes the members of one human group from another. ... Culture, in this sense, includes systems of values; and values are among the building blocks of culture.” (Hofstede 1980). Following the modelling of Scenario A, a tool for identifying cultural values (the Cultural Values Tool) was created and later tested through application in Scenario C. An electronic tool was created that enables various groupings within an organisation to identify the "as is" and "ideal" sets of cultural values.

The initial prototype consisted of a basic interface and focussed on two main areas: firstly a comparison of cultural values held by individuals, groups and the organisation itself, and secondly a comparison of 'current' with 'desired' positions for cultural values. For both areas the tool can be used to identify perspectives on cultural values at three different levels:

i. Individual: can provide own perspective on self, the group and the organisation within which s/he works

ii. Group: can provide the group perspective on the group itself and of the organisation within which the group operates

iii. Organisation: an organisational representative(s) can provide the organisational perspective

The 'comparison of cultural positions' section consists of eight cultural values or attributes, including their definitions, based loosely on the work of (Hofstede 1980) and (HampdenTurner and Trompennars 1994). The pairings are described in the following tables (figure $6)$;

\begin{tabular}{|l|l|}
\hline \multicolumn{1}{|c|}{ Universal } & \multicolumn{1}{c|}{ Particular } \\
\hline $\begin{array}{l}\text { Generic approach that is applicable to everything (e.g. } \\
\text { Lifecycle management applies to every programme } \\
\text { irrespective of size, value, purpose and local } \\
\text { circumstances). Formalisation and mandated } \\
\text { processes in all areas. }\end{array}$ & $\begin{array}{l}\text { Local characteristics prevail. Local rules and procedures } \\
\text { are created, over-riding mandated processes as necessary } \\
\text { to get to the goals more easily. }\end{array}$ \\
\hline
\end{tabular}

\begin{tabular}{|l|l|}
\hline \multicolumn{1}{|c|}{ Analysis } & \multicolumn{1}{c|}{ Synthesis } \\
\hline $\begin{array}{l}\text { 'The devil is in the detail'. Analytic approaches offer } \\
\text { the best hope for efficient management. Projects and } \\
\text { problems are decomposed in to individual elements for } \\
\text { simpler solutions. Managers demand facts, metrics, } \\
\text { and the 'bottom line'. }\end{array}$ & $\begin{array}{l}\text { The whole is greater than the sum of the parts. Emergent } \\
\text { behaviour is best handled by recognising patterns and } \\
\text { overall configurations. An holistic approach is important. }\end{array}$ \\
\hline
\end{tabular}

\begin{tabular}{|l|l|}
\hline \multicolumn{1}{|c|}{ Individualism } & \multicolumn{1}{c|}{ Communitarianism } \\
\hline $\begin{array}{l}\text { The needs of the individual come before the needs of } \\
\text { the organisation. Individual performance and ability } \\
\text { are encouraged, though a blame culture can arise. }\end{array}$ & $\begin{array}{l}\text { The needs of the organisation come before those of the } \\
\text { individual. }\end{array}$ \\
\hline
\end{tabular}

\begin{tabular}{|l|l|}
\hline \multicolumn{1}{|c|}{ Inner Directed } & \multicolumn{1}{c|}{ Outer Directed } \\
\hline $\begin{array}{l}\text { Internal/local values and perspectives are considered to } \\
\text { have more relevance than external opinion. } \\
\begin{array}{l}\text { Characteristics of 'self-starters' and self-motivated } \\
\text { individuals. }\end{array}\end{array}$ & $\begin{array}{l}\text { Extremely responsive to external influences (e.g. customer } \\
\text { demands, market trends, etc.). A characteristic of team- } \\
\text { based operations. }\end{array}$ \\
\hline
\end{tabular}




\begin{tabular}{|l|l|}
\hline \multicolumn{1}{|c|}{ Temporal Efficiency } & \multicolumn{1}{c|}{ Temporal Synchronicity } \\
\hline $\begin{array}{l}\text { Do things as fast as possible in the shortest possible } \\
\text { sequence of elapsed time e.g. time-based milestones } \\
\text { have over-riding priority. Efficiency is everything }\end{array}$ & $\begin{array}{l}\text { Synchronisation of effort within and across projects to } \\
\text { ensure co-ordinated maturity of engineering performance } \\
\text { and organisational learning. }\end{array}$ \\
\hline
\end{tabular}

\begin{tabular}{|l|l|}
\hline \multicolumn{1}{|c|}{ Power by Achievement } & \multicolumn{1}{c|}{ Power by status } \\
\hline $\begin{array}{l}\text { Influential positions/roles are held by individuals with } \\
\text { a record of past success; favouritism is rare. }\end{array}$ & $\begin{array}{l}\text { Influence is wielded by individuals with high personal } \\
\text { status in terms of seniority, qualifications, and experience. }\end{array}$ \\
\hline
\end{tabular}

\begin{tabular}{|l|l|}
\hline \multicolumn{1}{|c|}{ Low Power Distance } & \multicolumn{1}{c|}{ High Power Distance } \\
\hline $\begin{array}{l}\text { Decisions are made by those with the appropriate } \\
\text { knowledge and experience irrespective of role and are } \\
\text { filtered outwards in the organisation e.g. flat decision- } \\
\text { making systems, consensus-based decision-making }\end{array}$ & $\begin{array}{l}\text { Decisions emanate from above and are dispatched } \\
\text { downwards through the organisation e.g. hierarchical } \\
\text { decision-making systems }\end{array}$ \\
\hline
\end{tabular}

\begin{tabular}{|l|l|}
\hline \multicolumn{1}{|c|}{ High Risk Taking } & \multicolumn{1}{c|}{ Low Risk Taking } \\
\hline $\begin{array}{l}\text { Chaos and ambiguities are common, rules are there to } \\
\text { be broken or bent, risk taking is encouraged. }\end{array}$ & $\begin{array}{l}\text { Rules are there to be respected, desire or availability of } \\
\text { long-term stable careers, status quo is encouraged }\end{array}$ \\
\hline
\end{tabular}

\section{Figure 6. Cultural Value Pairings}

The 'comparison of 'current' with 'desired' positions' section provided the same eight cultural values, this time requesting that the user identify the 'current' and 'future' values for each of the required levels. In addition, a series of sub-questions are provided to place some context around the response.

The tool enables the organisation to firstly identify the cultural and subcultural values within, any differences between the levels of hierarchy (which may or may not be an issue depending on the activities that those roles are performing), differences in perspective that may be affecting the understanding of the way other individuals/groups work, and finally an idea of what the values should be. At each of the stages of data gathering, contextual information was provided in order to justify why certain values had been selected.

Enterprise Strategy. The term enterprise strategy refers to "the planned allocation of enterprise resources to achieve and maintain a configuration of the enterprise to meet the enterprise goals in a particular context or environment for the foreseeable future". This definition was created as an amalgamation of a number of definitions from (Katz 1970), (Galbraith 1978), and (Pascale \& Athos 1981).

Based on the definition above, the underlying purpose of enterprise strategy is to ensure that an enterprise will be able to achieve its overall goals within a given time period whilst surviving in its environment. And to do this by ensuring that the organisation configuration is "fit" to deliver this. (Kotelnikov 2005) expands this view of fitting an enterprise strategy to its environment by proposing that enterprise strategy should be a "stretch" exercise whereby the strategy (and in turn the organisational configuration) is dynamic and can be stretched to adjust with changes in the environment or the goals of the enterprise in order to survive. 
For enterprise strategy, there are three main questions to answer;

1. How does the environment/context affect the goals chosen?

2. What are the enterprise goals to be fulfilled?

3. How is the enterprise configured/resourced to fulfill these goals?

Every enterprise must create a top-level vision(s) statement based on an analysis of where the enterprise fits within its environment. Once this vision statement has been identified, the strategy can be decomposed into the missions that will be undertaken (the visible functions of the enterprise in terms of the products or services offered), according to the enterprise's policies ("the way we do things round here"). Each mission is broken down into a number of goals, for which tactics are defined for controlling how these goals are achieved. Finally the goals are broken down into actions that are constrained by the tactics. The last stage of creating the enterprise strategy is to identify what the enterprise configuration will be (i.e. how will the organisation be best placed to undertake the actions). There is a feedback loop from the configuration to the enterprise vision, as the configuration/resources may be limited and hence constrain the possible visions that are attainable (Figure 7).

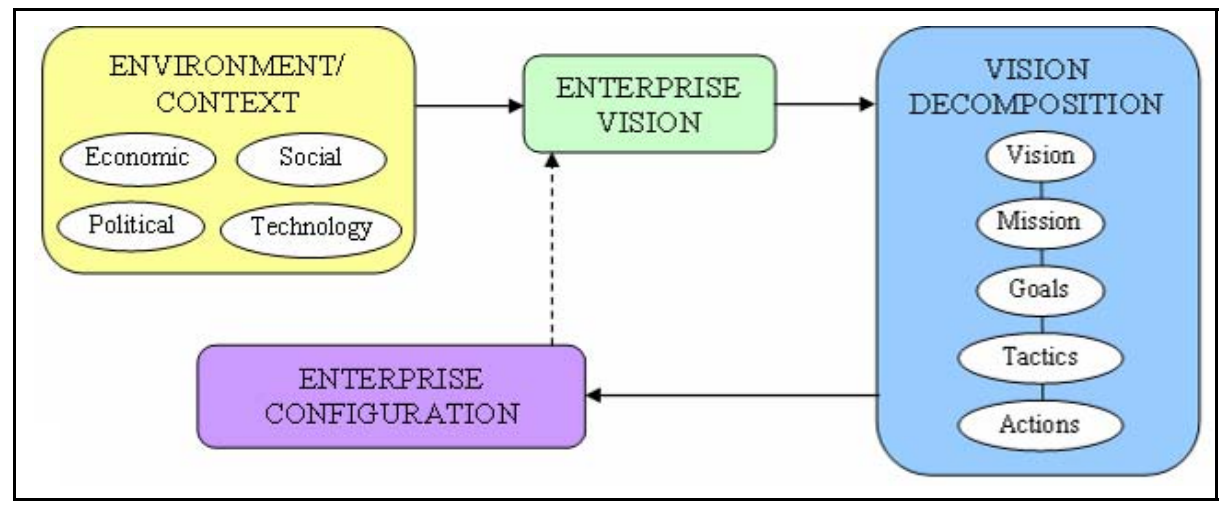

Figure 7. High Level Model of Enterprise Strategy 


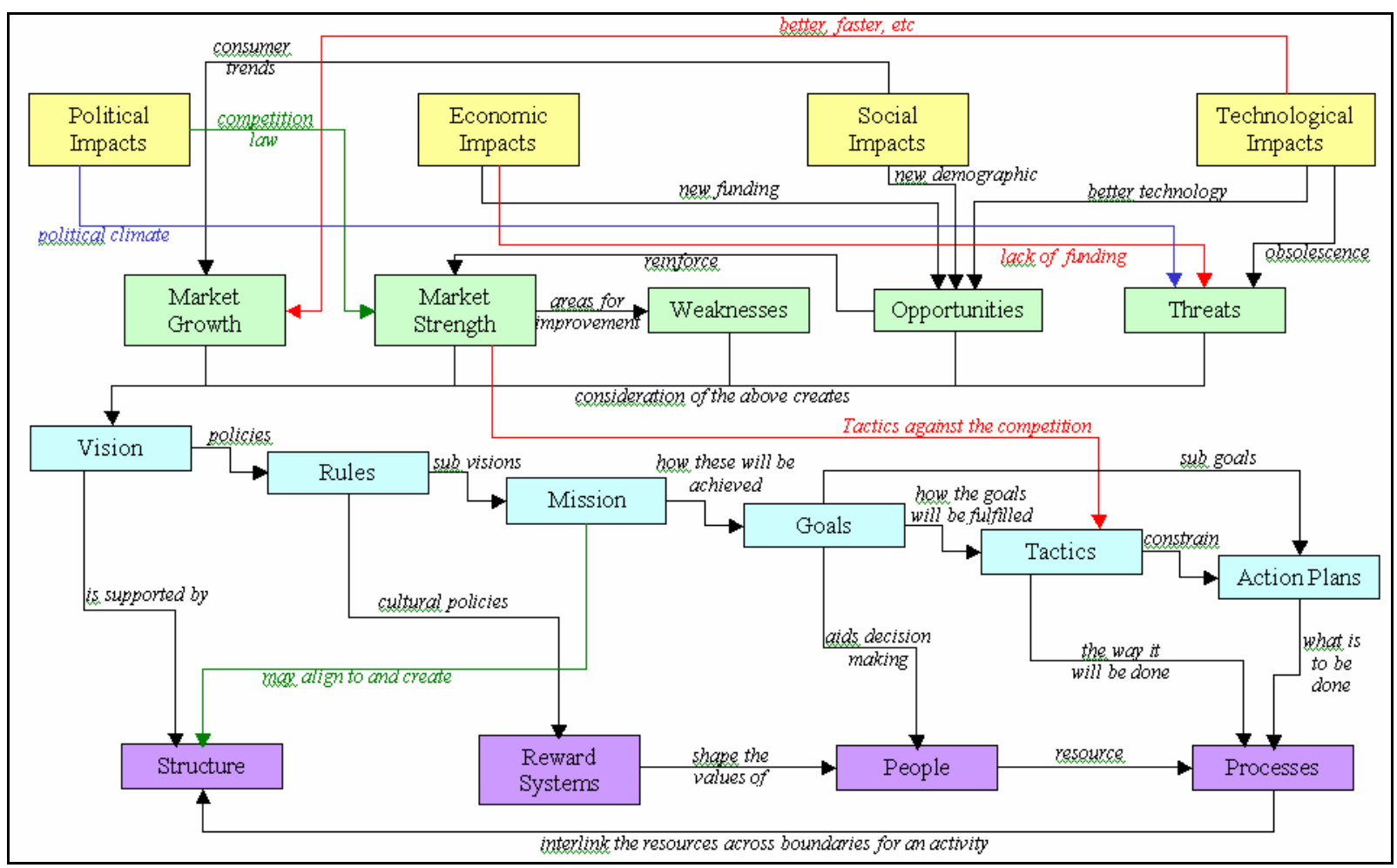

Figure 8. Generic Enterprise Strategy Model

Figure 8 illustrates a more detailed version of a generic model for enterprise strategy. Each of the four boxes in Figure 7 are broken down into component parts. It is not exhaustive in terms of the relationships between objects and it is not expected that every enterprise will have data to populate each box of the model (e.g. the vision may be decomposed in another way).

Decision Making Systems. Decision making systems (DMS) are a sub system of the overall enterprise system and comprise decision making agents (human and technological), processes, decision support technology, organisational structures, IT\&T infrastructures, etc. A DMS is a system for supporting the decision making made by a role - it does not include the inner cognitive decision making or psychological aspects of the decision maker.

The main tool used for the DMS modelling is the GRAI grid and GRAI net (Doumeingts et al. 1992a). The GRAI technique was developed by the University of Bordeaux and is grounded in General Systems Theory (Le Moigne 1977).

Figure 9. GRAI Grid GRAI grids (Figure 9) are used to describe the decision making structures and are based on decision levels; how long a decision is valid for $(\mathrm{H})$ and the period when decisions must be reconsidered $(\mathrm{P})$. They can be

\begin{tabular}{|c|c|c|c|c|c|c|}
\hline & \multicolumn{6}{|c|}{ Functions } \\
\hline & & $\begin{array}{c}\text { External } \\
\text { Information }\end{array}$ & $\begin{array}{c}\text { To manage } \\
\text { orders }\end{array}$ & To plan & $\begin{array}{l}\text { To manage } \\
\text { resources }\end{array}$ & $\begin{array}{c}\text { Internal } \\
\text { Information }\end{array}$ \\
\hline & $\begin{array}{l}H_{n}= \\
P_{n}=\end{array}$ & & & $\Downarrow$ & & \\
\hline & $H_{n-1}=$ & - & & 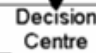 & & - \\
\hline & $\begin{array}{l}H_{1}= \\
P_{i}=\end{array}$ & & $\checkmark$ & $\Downarrow$ & 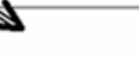 & \\
\hline & $\begin{array}{l}H_{0}= \\
P_{0}=\end{array}$ & & & & & \\
\hline
\end{tabular}
used to identify what the decision hierarchies/dependencies are and what information is 
required for each decision. The GRAI grid can be used for identifying Strategic, Operational or Administrative decision making centres (strategic will tend to be the higher decision levels of the GRAI grid, operational and administrative will tend to be lower levels).

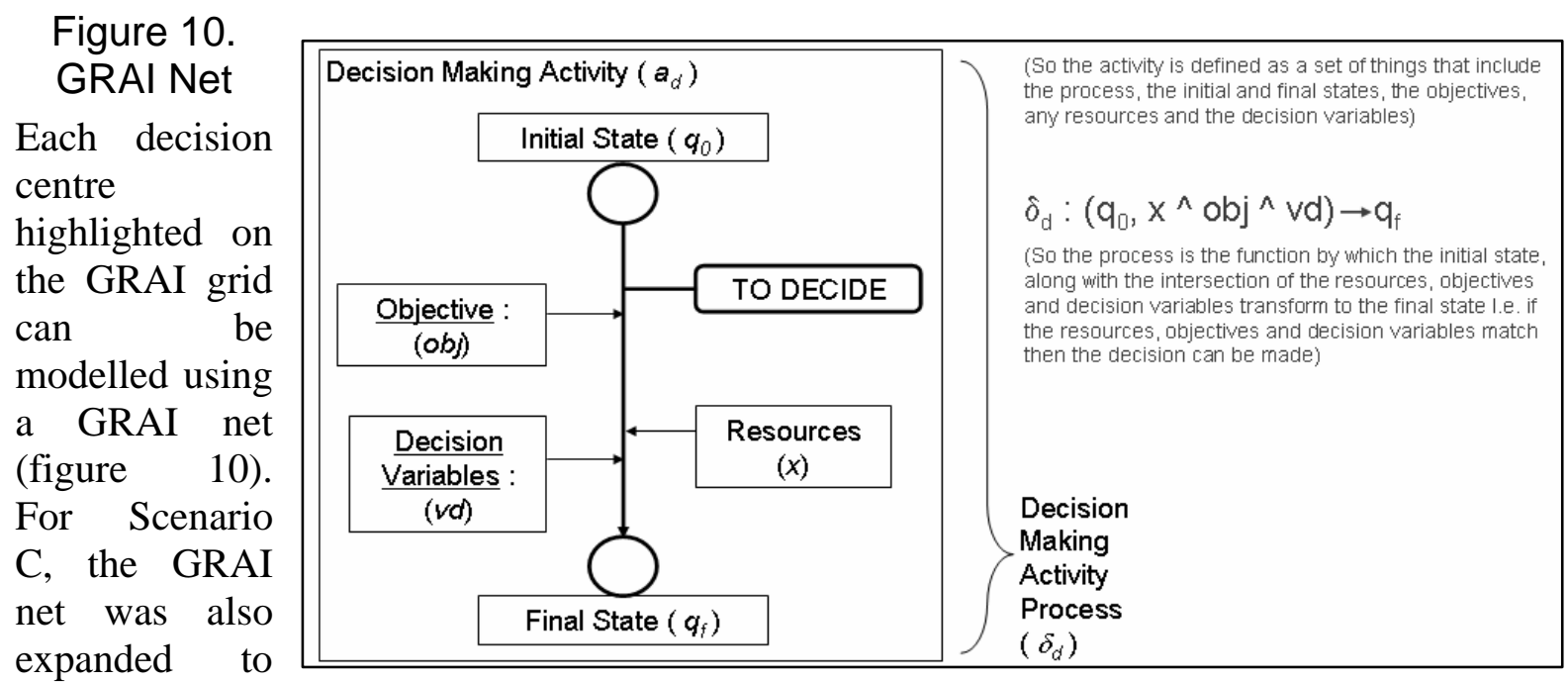

include an area labeled constraints with a view to anticipating the impact on other enterprise system objects and vice versa (e.g. the strategy of the enterprise will constrain the decision being made).

Competency Modelling. Role holders need to have sufficient knowledge (both tacit and formal) to carry out the tasks allocated to them. Knowledge in this sense is interchangeable with the term competency that seems to be favoured in the literature.

The Competency Tool is based on work developed as part of the SIMPLOFI project undertaken at Loughborough University (Sinclair 1997). Competency is described as the level of skill and experience that a role holder requires in using certain knowledge types. Figure 11 shows a brief diagram of the top levels of the knowledge tree developed during previous projects and demonstrates how the knowledge nodes decompose. For each role at each stage of the activity in the functional modelling, some form of competency is required. This knowledge is also categorised into four different levels - "know about", "entry level", "experienced" and "expert". The level of knowledge required can be related either to the level required by any role holder to carry out a designated activity or could relate to the personal background of the individual role holder. 


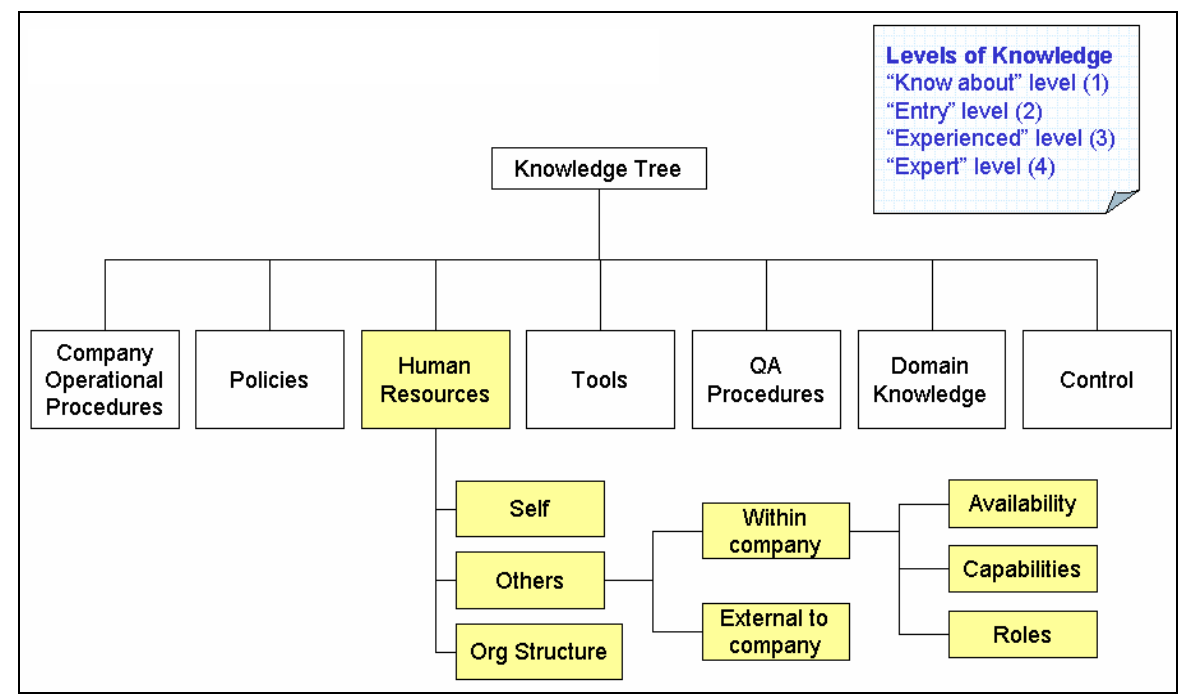

Figure 11: Knowledge Tree

The knowledge tree (basic taxonomy of knowledge classes and subclasses within it) has over 400 separate nodes and initially covered all aspects of knowledge required for a manufacturing plant. For Scenario $\mathrm{C}$ the domain knowledge branch of the tree was expanded and tailored to the organisation (i.e. included research skills and specific knowledge in the research field).

(Sinclair 1997) also outlines grouping and stopping rules for role allocation based on the competencies for each activity. Due to the brevity of the paper it is not possible to go into great detail on these rules. The grouping rules are based on whether the same knowledge classes are being used and the availability of the role in terms of the sequence of activities. The stopping rules define when grouping should stop, e.g. when a role is expected to be at level 4 (expert/specialist level) in too many areas. For Scenario C the competency tool was used to identify the competency requirements for a subset of role holders undertaking specific activities. The grouping rules could then be used to ascertain whether the role allocation could be improved. The stopping rules were applied to identify whether there were any unreasonable demands on a role.

\section{Towards an Integrated Model of the Enterprise System}

One of the outputs of the VORTICS project was to assess whether it would be feasible to integrate the more traditional modelling views (function, information and resource) with the softer organisational aspects. Where traditionally the organisational view has consisted primarily of structure and role (or job title) in a company, it has been expanded to take into account the enterprise strategy, cultural values, competencies, roles (in terms of the actual nature of involvement in an activity) and decision making. It is important to understand that within the context of this paper the term integration is used to describe the understanding of, combination, and manipulation of the traditional and soft models (through consideration of the interactions between them). It is not integration in terms of how the enterprise is supported seamlessly through IT support infrastructure (more commonly coined "Enterprise Integration”). As explained in earlier sections each of the views are being considered as 
systems within the enterprise systems-of-systems. Each have been used independently, however there are overlaps between the models and coupling between the different systems.

In order to integrate the systems together, the components of each model were considered in turn and different interactions identified. With the components or building blocks of an enterprise system already determined earlier in the paper, an $\mathrm{N}^{2}$ diagram/matrix was created by first brainstorming within a group of researchers. The $\mathrm{N}^{2}$ diagram (figure 12) shows the envisaged links between each component (this is done in terms of two-way relationships; i.e. the relationship between process and role is not necessarily the same as role to process). Each relationship was given a weighting depending on the strength of the link; " 5 " meaning that the components are the same item or part of each other; " 4 " is a strong, direct link; " 3 " is a weak, direct link; " 2 " is an indirect link; " 1 ” is a link but only in limited circumstances. For each link comments were used to provide traceability by recording and justifying why the weighting was given. The $\mathrm{N}^{2}$ diagram only considers first order relationships, not compound relationships. Each cell only considers one relationship (so multiple relationships between two components are not addressed). It can be seen that there are many interactions between the different components and hence the enterprise system could be viewed as being a tightly coupled, complex system. The $\mathrm{N}^{2}$ diagram enables the researcher to identify, explore and record the relationships between the enterprise components.

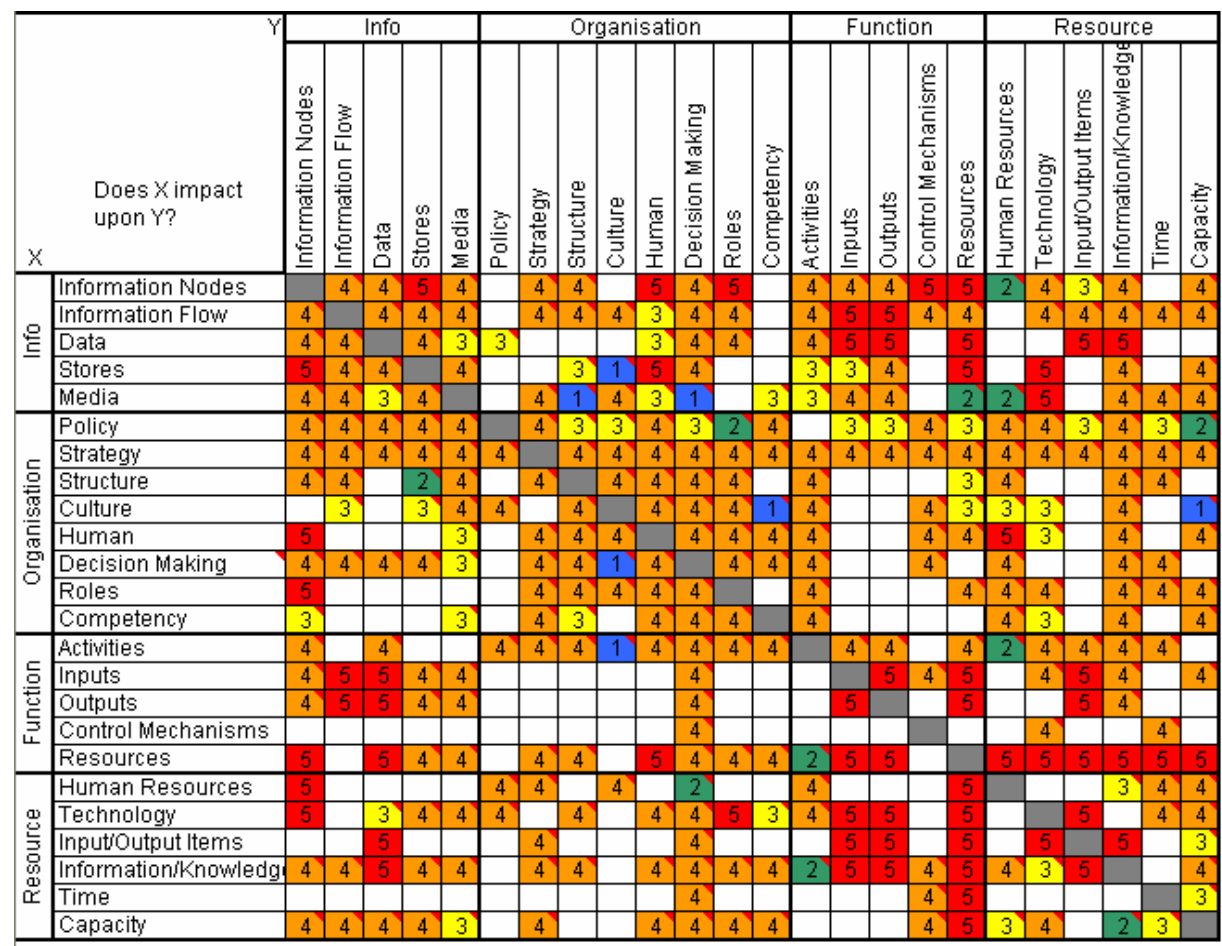

Figure 12. $\mathrm{N}^{2}$ Matrix of Enterprise Component Interactions

From further literature review a number of other links were identified. Undertaking the Scenarios also uncovered more plausible links. In order to manage the data being gathered a simple database was constructed which contained information about the link, the relationship and the context (situations under which the link is valid). 
There are a multitude of enterprise architecture frameworks that cover to different extents the different components in an enterprise, however there are currently no architecture frameworks that consider or include the soft enterprise characteristic models in wide usage. Rather than reinvent the wheel, it was decided to use an existing framework as a basis for extension. Work undertaken as part of the Unified Enterprise Modelling Language (UEML) project (Doumeingts 2003b) developed a class diagram showing objects within an enterprise and the relationships between them (the purpose of the UEML was to enable enterprises to interoperate and share models). As part of the VORTICS project, the UEML class diagram was further extended to contain the additional soft enterprise objects and additional relationships recorded in the database. For clarity, only the relationships that apply to generic enterprises were included in the integrated enterprise system model (figure 13). There are a number of other frameworks that could have been used as a baseline for extension; examples are ARIS (Scheer, 1994), MoDAF (MoDAF Partners, 2005), DoDAF (DoDAF, 2003), CIMOSA (Amice Consortium, 1993), PERA (Williams, 1994), and TOGAF (The Open Group, 2002). It was decided that UEML was to be used as a vehicle for expansion because it was not specific to any particular industry or domain and also had overlaps with the types of models that had already been used to model the scenarios (in particular GRAI was one of the three mechanisms incorporated into UEML).

The Integrated Enterprise Model shows how the different components within the different system models are brought together. A good example of how different views are integrated into the model is to look more closely at the role model.

- The role model interacts with the functional model (the functional model being the set of activities, their inputs, outputs, controls and mechanisms) as the roles are involved in enabling the activities to be undertaken.

- The role model also interacts with the competency model as a role holder requires a certain level of competency in order to perform the activities assigned to it.

- The role model interacts with the organisational structure model as the roles do not perform in isolation but are positioned in hierarchies of responsibility during different activities. Role holders may also be individuals within a structure, or may be grouped together to form a role for a group.

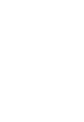




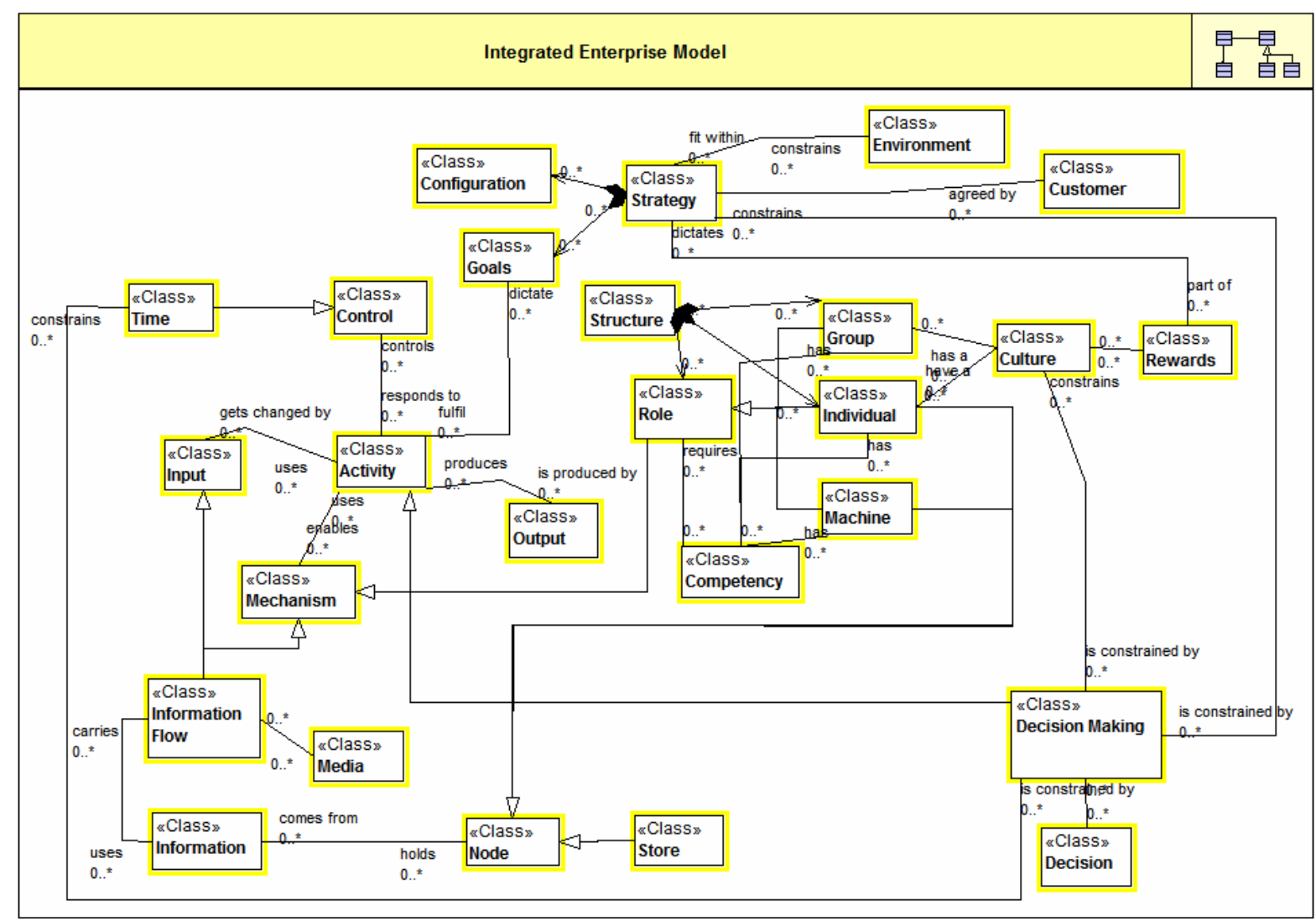

Figure 13. Integrated Model of an Enterprise System

Finally, the integrated model was tested and evaluated through instantiating the objects using the models created as part of the Scenario C. The integrated model was subsequently refined as models were populated. Both the integrated model of Scenario C (figure 14) and the integrated enterprise system model (figure 13) were modelled and stored in a piece of software by Troux called Metis ${ }^{\mathrm{TM}}$ which enables the designer to create model views (through either use of built in templates or creation of new generic object types). Metis ${ }^{\mathrm{TM}}$ allows the user to traverse easily around the different individual models and link different types of model together.

Figure 14 shows a screen-grab of the overall integrated model for Scenario C. Each folder represents a model view - some of the interactions are shown as direct lines between objects both within and external to a folder. As would be expected, the integrated model is complex in that it has many nodes and interactions. Within Metis ${ }^{\mathrm{TM}}$, in order to make the models less messy for viewing, "tunnel throughs" can be created whereby an existing object may be put into other views without creating another separate object. These tunnel throughs are represented by yellow outlines. If all the hidden relationships were shown on the diagram it would be an indecipherable mesh of lines. Although the integrated model for Scenario C will not analyse or provide direct answers to enterprise wide questions, it does enable the user to not only see the effect within one model, but also to follow through the relationships into other models so the user can assess the impact. Traceability issues between the individual 
standalone models were highlighted during integration, corrected and revalidated with the scenario stakeholders.

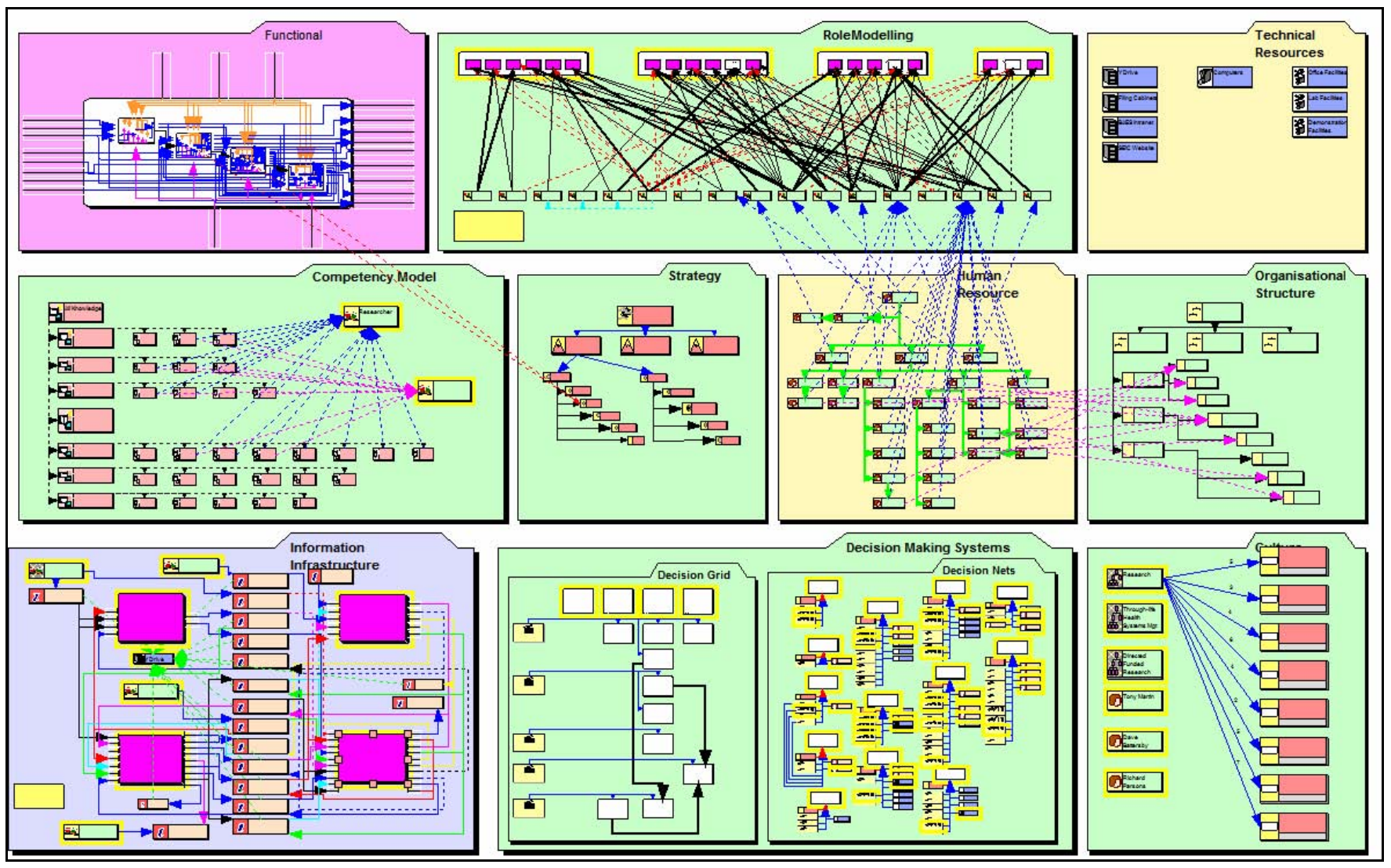

Figure 14. Scenario C Integrated Model

In Scenario $\mathrm{C}$ a number of techniques and models were used. Because the integrated enterprise system model is abstract;

- it is independent of modelling languages when creating an instantiation of the model

- the objects may be utilized in different groupings and hence different views or boundaries of the systems can be used

- different models of enterprises may have a better likelihood of integrating as the integrated enterprise system model acts as a common denominator

Not every object within the integrated enterprise system model needs to be considered within the models being created, as explained in previous sections the modelling should be fit for purpose.

\section{Conclusions}

This paper has introduced first how the enterprise may be viewed as a system-of-systems. From this premise a number of new systems (or modelling views) were developed that fulfilled gaps in current enterprise modelling capability. Finally an integrated model of the enterprise system was created including both the existing and new models. 
The underlying purpose for modelling enterprises is ultimately to increase performance and increase profits (or reduce costs!). Through the modelling of the scenarios the value of enterprise modelling became more apparent. Benefits included:

- provision of a representation/visualisation for the enterprise to capture information and provide a common basis for discussion

- provision of a formal baseline set of models that can be updated and utilized as the enterprise changes

- deeper understanding of the inner workings of the enterprise

- provision of insight into problems

- diagnosis of symptoms of problems

- increased transparency for members of the organisation

- comparison of viewpoints of those involved

- analysis the enterprise

- identification of improvements

- looking at alternatives and the possible impacts of making changes

- planning for the future

The integrated model is by no means a complete resource, but a starting point (hence the title of this paper, "towards...."). There are a number of limitations with the integrated model to be aware of. Although it has been validated through application on Scenario C it needs further instantiations of different types of scenarios and subsequent refinements. There are limitations in terms of the dynamicism of the model, in order to adhere to the GERA the enterprise lifecycle dimension must also be explored. The models are purely qualitative at present, in order to provide meaningful performance metrics some (if not all) the models will have to have a quantitative nature to simulate. At present the integrated model only contains a specified set of generic relationships and is hence not context based.

Within the bounds of the VORTICS project, the integrated model will be used to drive the initial specification for the VORTICS engine itself. Because the integrated model is abstract it will be able to be used as a translation tool such that most enterprise models can be described in such a way that the VORTICS engine/intelligence would be able to understand. The VORTICS project ended in late 2006, it has been superceded by the Unified Modelling of Complex Enterprise Systems (UMCES) project which will expand on the work of VORTICS to try and identify the complexity issues with an integrated enterprise model, in addition to driving forwards in terms of being able to simulate parts of the model. At the same time a new set of scenarios within other industries has been identified and will be used to improve and refine the integrated model.

\section{References}

Amice Consortium CIMOSA: open system architecture for CIM, Springer, 1993 Callan et al, “The Role Matrix Technique”, INCOSE Proceedings, July 2005

Chen D. \& Vernadat F., "Standards on Enterprise Integration and Engineering - State of the Art”, Int. J. Computer Integrated Manufacturing Vol 17 No. 3 Pgs 235-253, 2004

Department of Defense “Department of Defense Architecture Framework” Version 1.0, 2003

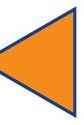


Doumeingts G. et al., "GIM, GRAI Integrated Methodology - A methodology for designing CIM systems”, University of Bordeaux report , 1992

Doumeingts G., "UEML Project - Final Review of UEML Project”, presentation at UEML Final Review Meeting - Brussels, June 2003

Galbraith J.R. \& Kazanjian R.K., Strategy Implementation - Structure, Systems and Process, $2^{\text {nd }}$ edition. West Series of Strategic Management, 1978

Hampden-Turner C. \& Trompennars F., The Seven Cultures of Capitalism, London, Piatkus, 1994

Hofstede G., Culture's Consequences: International Differences in Work Related Values, Beverley Hills, CA, Sage Publications, 1980

IFIP/IFAC Task Force, "GERAM: Generic Enterprise Reference Architecture Methodology”, Version 1.6.2 ISO15704 Annex A, 2000

Katz R., Management of the Total Enterprise, Prentice-Hall, 1970

Kotelnikov V., "Mastering Your Enterprise Strategy”, Innovation Unlimited. Available online at http://Www.1000ventures.com/business_guide/mgmt_inex_stategy.html, 2005

Le Moigne, J. L., La Théorie du Système Général, Presses Universitaires de France, 1977

MoDAF Partners, "MoDAF Technical Handbook” Version 1.0, MODAF-M07-022, 2005

NIST, “Integrated Definition for Function Modelling”, FIPS publication 183, 1993

Pascale R. \& Athos A., The Art of Japanese Management, Simon \& Schuster, 1981

Scheer A.W., Business process engineering: reference models for industrial enterprises. Springer, 1994

Sinclair M.A., “A Description of the Knowledge Network - Final Report”, HRI97/14 HUSAT report, 1997

The Open Group The Open Group Architecture Framework Version 8.0. The Open Group (available online at http://www.opengroup.org/architecture/togaf/), 2002

Vernadat F.B., Enterprise Modelling and Integration - Principles and Applications, Chapman and Hall, London, 1996.

Williams, T.J. The Purdue Enterprise Reference Architecture Computers in Industry, 24(2), 141158, 1994

\section{Biography}

Grace Kennedy (née Ng) graduated with a Masters in Systems Engineering with Diploma in Industrial Studies from Loughborough University in 2002. Since graduation she has been a Research Associate also at Loughborough University. She has previously researched into Systems Engineering in the wider world, Human Reliability Assessment (HRA) in the military domain, and more recently Organisational Systems Engineering. Her current project is researching into the development of a unified model for complex engineering systems and is undertaking a part time $\mathrm{PhD}$ in the enterprise modelling field. Grace is a member of Institution of Engineering and Technology (IET).

Carys Siemieniuch is a Senior Lecturer in the Dept of Electronic \& Electrical Engineering at Loughborough University. A systems ergonomist for 17 years, with both UK professional and European CREE registration, she has expertise across the full range of systems-related human factors topics, but retains a particular interest in knowledge life cycle management systems, 'soft' aspects of enterprise modelling techniques and individual/team situational awareness/decision making structures and processes. She has served as the Ergonomics 
Society representative to two UK Foresight panels: Defence \& Aerospace and Manufacturing and is a registered EU 'expert' with evaluation, project reviewer and project management expertise within the Framework Programmes.

Murray Sinclair is a Senior Lecturer in the Department of Human Sciences. Current research areas concern advanced manufacturing technology e.g. human supervision and control in 'flexible' manufacturing environments, knowledge structures and the roles of knowledge within organisations and structures for engineering governance. He is a member of standards committees CEN/TC310/WG4 Advanced Manufacturing Technology, CEN/TC122 Ad Hoc Working Group on co-ordination of Ergonomics standards, and BSI PH/9/6 User System Interfaces. Murray is a Fellow and Council Member of the Ergonomics Society, a registered European Ergonomist and member of the IEEE Engineering Management Society. 